\title{
ACEPTACIÓN/RECHAZO SOCIAL INFANTIL: RELACIÓN CON PROBLEMAS EMOCIONALES E INTELIGENCIA EMOCIONAL
}

\section{Child social rejection/acceptance: relationship with emotional problems and emotional intelligence}

\author{
Ornela Mateu-Martínez ${ }^{1}$, José Antonio Piqueras ${ }^{2}$, María Rivera-Riquelme ${ }^{2}$ \\ José Pedro Espada², Mireia Orgilés ${ }^{2}$
}

\begin{abstract}
Resumen
El objetivo del estudio fue analizar la relación de la aceptación/rechazo social con la sintomatología ansioso-depresiva y la inteligencia emocional en niños españoles de 8 a 12 años. La muestra estuvo compuesta por 94 alumnos $(45.7 \%$ varones, edad media $=9.76 ; D T=$ 1.09). Los resultados indicaron que el rechazo social se relaciona con la sintomatología de fobia social, disforia y baja autoestima, mientras que la aceptación social se relaciona con habilidades sociales. En resumen, el rechazo social se relaciona con problemas emocionales, mientras que la aceptación social lo hace con inteligencia emocional. Este estudio sugiere la importancia de desarrollar programas de competencias socio-emocionales para promover la aceptación social y prevenir los trastornos emocionales asociados al rechazo social en la infancia.
\end{abstract}

Palabras clave: Rechazo social, aceptación social, inteligencia emocional, ansiedad, depresión, niños.

\begin{abstract}
The aim of this study was to analyse the relationship of social acceptance/rejection whit anxietydepressive symptomatology and emotional intelligence in Spanish children. The sample consisted of 94 students of Elementary School between 8 and 12 years. Results revelled the significant relationship between rejection index and symptomatology of social phobia, dysphoria and low self-stem, while the model for choices index is related whit social skills. In resume, social rejection is related whit emotional problems, whereas social acceptance does with emotional intelligence. This study suggests the relevance of developing socio-emotional competences programs to promote social acceptance and to prevent emotional disorders related with social rejection in childhood.
\end{abstract}

Keywords: Social rejection, social acceptance, emotional intelligence, anxiety, depression, children.

1. Universidad del Sur, Cancún, México.

2. José Antonio Piqueras. Docente Departamento de Psicología de la Salud, Universidad Miguel Hernández de Elche. 03202 - Elche. jpiqueras@umh.es 
En opinión de Becerra, Godoy, Véjar y Vidal (2013) la necesidad de aceptación social se entiende como la función de agradar y pertenecer a un grupo social, que es una de las necesidades básicas del ser humano.Los niños son un colectivo de especial atención respecto a esto, ya que en la escuela este fenómeno se traduce en la necesidad de estima y aprobación social, de establecer de relaciones de amistad y de vínculos afectivos con los iguales.

La importancia de gozar de un adecuado grado de aceptación social y habilidades sociales para establecer buenas relaciones dentro de un grupo es cada vez más reconocida por los investigadores, otorgando una relevancia equiparable a la del desarrollo de una alta capacidad académica o intelectual (Goleman, 1996). Un importante indicador de ajuste para el bienestar de un niño es su habilidad para establecer relaciones satisfactorias con sus iguales, sobre todo en el momento de la escolarización en que el niño pasa del principal sistema de socialización, el hogar, al siguiente sistema socializador, la escuela, donde amplía su red de contactos y empieza a ser influido por el comportamiento de sus pares. Morrison y Masten (1991) sugieren que el desarrollo de la autoestima, la estabilidad emocional y la capacidad para relacionarse con los demás están relacionadas con la calidad de las relaciones sociales que se establecen a edades tempranas, y tiene un gran impacto en la adolescencia y la edad adulta de la persona.

Sin embargo, investigaciones previas informan que desde hace más de medio siglo se producen estos fenómenos de discriminación entre pares que truncan esa necesidad universal de aceptación social (Díaz-Aguado, 2005). En las aulas se observa una distinción frecuente entre los alumnos que son aceptados socialmente y aquellos que son rechazados por sus iguales. Diversas investigaciones se han interesado en estudiar fenómenos relacionados con procesos de discriminación tales como rechazo social, rechazo entre iguales, bullying, acoso, aislamiento, violencia, maltrato, exclusión o marginación, y hacer una caracterización de las variables con las que se relacionan (Jaramillo, Díaz, Niño y Tavera, 2006).

En la actualidad, las conductas de rechazo social han ido transformándose desde formas abiertamente hostiles y manifiestas hasta formas más sutiles y encubiertas (Becerra et al., 2013). Opinan que el rechazo social se origina en la expresión de un "no me gustas" por un número significativo de compañeros, que conlleva a que se le ignore, evite o aísle de la interacción social propia de la vida escolar, excluyéndolo de las oportunidades de aprendizaje y de diversión con sus pares. El rechazo social puede manifestarse de forma activa, por ejemplo mediante conductas como la ridiculización, las ofensas o las burlas, o puede manifestarse de forma pasiva mediante conductas indirectas tales como ignorar o desestimar (García-Bacete et al., 2013).

El rechazo social es un proceso muy relacionado con los grupos, y actualmente puede encontrarse este fenómeno en todas las aulas (Moreno, Muñoz, Pérez y Sánchez, 2004). España se encuentra entre los cinco países europeos con mayor frecuencia de víctimas de bullying, con una probabilidad entre un $22 \%$ y un $23 \%$, lo cual se sitúa por encima de la media europea (Analitis et al., 2009). En el estudio de García-Bacete et al. (2008) se refleja que aproximadamente 1 de cada 10 alumnos son rechazados por sus compañeros, datos similares a otras investigaciones nacionales e internacionales. Investigaciones recientes han confirmado que un 14\% de alumnos en el primer curso de Educación Primaria son rechazados, convirtiéndose aproximadamente la mitad de ellos en niños rechazados crónicos (Marande, 2011). A pesar de que el rechazo social es muy frecuente en los centro escolares, la información disponible para comprender sus orígenes y contrarrestarlo eficazmente es muy escasa (Jaramillo et al., 2006).

Respecto a su prevalencia, hay estudios que revelan que el rango de edad en el que existe mayor riesgo de sufrir rechazo social en forma de bullying se da entre los 9 y los 15 años (Seals y Young, 2003). Si bien se asume que en los contextos escolares la manifestación de aceptación y rechazo social son, en parte, inevitables en la vida escolar, cabe reconocer que el rechazo social puede convertirse en un problema cuando es percibido como prolongado o consistente al provocar varias consecuencias psicosociales en la adaptación, tanto actual como a largo plazo, de los niños maltratados y/o rechazados (Malinosky-Rummel y Hansen, 1993) 
Gifford-Smith y Brownell (2003) señalan que las graves consecuencias relacionadas con el rechazo social manifiestan que los alumnos rechazados por sus compañeros son el grupo sociométrico con más riesgo. Los estudios coindicen en que no existe un perfil del alumno rechazado, aunque se han encontrado unos patrones de conducta comunes en la mayoría de ellos: a) menos conductas prosociales; b) más comportamientos delictivos, agresivos o disruptivos; c) falta de atención y más conductas inmaduras; d) más ansiedad y evitación; e) poca actividad social; f) baja autoestima, autoconcepto negativo, inseguridad y depresión; g) insatisfacción con las relaciones con los compañeros y profesores, dificultades de aprendizaje, mayores posibilidades de mal rendimiento académico y abandono escolar; h) más sensibilidad a ser rechazados posteriormente y mayor riesgo de desarrollar problemas psiquiátricos en la adultez (Bierman, 2004; García-Bacete, Monjas y Sureda, 2008; Plazas et al., 2010; Estévez, Martínez y Jiménez, 2009). Así, puede decirse que entre las víctimas de rechazo social predominan los problemas de ansiedad (Albores-Gallo et al., 2011).

Por otra parte, las investigaciones de los últimos años han destacado el auge de la inteligencia emocional, otorgando mayor importancia a las competencias socio-emocionales, que determinan el establecimiento de un alto grado de empatía y vinculación con otras personas. Se deduce que los alumnos que alcancen a un nivel elevado en el dominio de estas competencias establecerá y mantendrá más lazos afectivos con los demás durante la etapa escolar, mientras que aquellos que no consigan desarrollarlas presentarán más dificultades de adaptación y posibilidad de sufrir rechazo social (Fernández y Extremera, 2002). En consecuencia, la empatía es considerada como uno de los aspectos principales de la competencia socioemocional, y un factor fundamental de protección contra la aparición de conductas antisociales (Mestre, Samper y Frías, 2004).

A pesar de todos estos datos, no existen estudios que hayan examinado la relación entre rechazo/ aceptación social y síntomas emocionales e inteligencia emocional conjuntamente en niños. En consecuencia, este trabajo trata responder a la siguiente pregunta de investigación: ¿en qué medida se relacionan el rechazo social y/o la aceptación social con la sintomatología ansioso-depresiva y la inteligencia emocional en niños españoles? Para ello, la población destino fueron niños españoles de 8 a 12 años, puesto que el rechazo social más acusado en forma de acoso se produce en edades muy tempranas (entre 8 y 11 años) (Analitis et al., 2009).

En concreto, las hipótesis de este trabajo son: (1) esperamos que mayores índices de rechazo (rechazo social) se asociarán con mayor sintomatología ansiosa y depresiva; (2) mayores índices de rechazo con puntuaciones más bajas en las dimensiones de inteligencia emocional; (3) mayores índices de elecciones (aceptación social) con menor sintomatología emocional; (4) mayores índices de elecciones presentarán mayores puntuaciones en las dimensiones de inteligencia emocional.

\section{MÉTODO}

\section{Participantes}

La muestra total de participantes estuvo compuesta por 94 estudiantes $(45.7 \%$ varones, edad media $=9.76$; DT $=1.09)$ de Escuelas Públicas de Educación Primaria $\left(3^{\circ}, 4^{\circ}, 5^{\circ}\right.$ y $6^{\circ}$ curso $)$ de la Provincia de Alicante, con edades comprendidas entre los 8 y 12 años.

El tipo de muestreo fue de naturaleza intencional o de conveniencia. Los criterios de inclusión que se contemplaron para la selección de los centros educativos que comprenden la muestra fueron: ser una escuela de educación primaria ubicada en la provincia de Alicante y haber obtenido la autorización del equipo directivo escolar para participar en la investigación. Los criterios de inclusión que se utilizaron para la selección de alumnos fueron: estar cursando segundo o tercer ciclo de Educación Primaria, y haber obtenido el consentimiento por escrito de los padres.

\section{Instrumentos}

Para evaluar el rechazo social se utilizaron dos cuestionarios sociométricosen función del cicloescolar: para el segundo ciclo de Educación Primaria se utilizó el «Cuestionario Sociométrico para Niños (CSN)» (de 6 a 10 años) y el tercer ciclo (de 11 a 13 años) se evaluó mediante el «Cuestionario Sociométrico para 
Preadolescentes (CSP)», ambos elaborados por DíazAguado (1995). El método que utiliza se basa en las nominaciones como un reflejo de la integración social de cada alumno en su respectiva aula. La puntuación obtenida se refleja por un lado el rechazo social, que se asocia con el índice de rechazos obtenido a través del número posible de rechazos y el total de alumnos de cada aula. Por otro lado, la aceptación social se asocia al índice de elecciones, que se obtiene de la misma forma que el anterior pero mediante el número de elecciones. Su aplicación dura aproximadamente 5 minutos.

La inteligencia emocional se valoró mediante el «Test de Inteligencia Social Emocional» diseñado por Chiriboga y Franco (2001). Se compone de 60 ítems, distribuidos por igual en cinco escalas: autoconciencia, autocontrol, automotivación, empatía y habilidad social. La prueba ofrece una sensibilidad del $45.45 \%$ y una especificidad del $75.95 \%$, y sus valores predictivos son $34.48 \%$ positivo y $83.33 \%$ negativo, con un $69.1 \%$ de valor predictivo global. El tiempo estimado de aplicación es de 30 minutos. Posee una escala de respuesta tipo Likert de 0 a 3, sugiriendo que a mayor puntuación, mayor Inteligencia Emocional presenta el individuo. Para esta muestra, los valores alfa de Cronbach fueron: puntuación total $=.83$, Autoconciencia $=.53$, Autocontrol $=.54$, Automotivación $=.62$, Empatía $=.63$, Habilidad social $=.71)$.

Para evaluar la sintomatología depresiva se utilizó la versión española del «Inventario de Depresión Infantil» (Children's Depresión Inventory; CDI, Kovacs, 1992; versión española de Del Barrio, Moreno-Rosset y López-Martínez, 1999). Se compone de 27 ítems distribuidos en dos escalas: Disforia y Autoestima. Se estima un tiempo medio de 10 minutos para su aplicación. Posee una escala de respuesta tipo Likert de 0 a 2, sugiriendo que a mayor puntuación, mayor sintomatología depresiva presenta el individuo. Es una prueba validada para población española (Figueras, Amador-Campos, Gómez-Benito y Gándara, 2010). Las puntuaciones de nuestro estudio reflejaron los siguientes valores de consistencia interna $($ alpha de Cronbach $):$ Disforia $=.80$, Autoestima $=.68$ y puntuación total $=.84$ ).
Para valorar la sintomatología ansiosa, se utilizó la versión española europea de la «Escala de Ansiedad Infantil de Spence» (Spence Children's Anxiety Scale; SCAS, Spence, 1998; versión española europea de Orgilés, Méndez, Spence, Huedo-Medina y Espada, 2012). Se compone de 6 escalas que engloban 45 ítems: Ansiedad por separación, Fobia social, Trastorno obsesivo-compulsivo, Pánico/Agorafobia, Miedo al daño físico y Ansiedad generalizada. Se estima un tiempo de aplicación de 30 minutos aproximadamente. Posee una escala de respuesta tipo Likert de 0 a 3 , sugiriendo que a mayor puntuación, mayor sintomatología ansiosa presenta el individuo. En esta muestra se obtienen los siguientes valores alfa de Cronbach: Trastorno de Ansiedad Separación $=.58$, Fobia Social $=.69$, Trastorno Obsesivo-compulsivo $=.56$, Pánico/Agorafobia $=.80$, Daño físico $=.54$, Trastorno de Ansiedad Generalizada = .62 y puntuación total $=.86$. Posee unas propiedades psicométricas satisfactorias con población española, presentando alta consistencia interna (alpha de Cronbach $=.89$ ) y validez convergente $(r=.41)$ adecuada con otras medidas de ansiedad.

\section{Procedimiento}

Se llevó a cabo un diseño no experimental de tipo transversal correlacional. Las variables dependientes fueron el índice de rechazos y el índice de elecciones (relación entre el número de rechazos y elecciones posibles y el total de alumnos, respectivamente). Las variables independientes incluyeron las dimensiones de ansiedad y depresión e inteligencia emocional.

El diseño y ejecución de la investigación se ajustó a las normas éticas nacionales e internacionales establecidas para la investigación científica. El manejo de los datos se hizo de manera confidencial, ya que no se realizaron análisis individuales (Martínez et al., 2014).

En un primer momento, se realizó una entrevista con los equipos directivos de los centros escolares que accedieron a participar para exponer los objetivos de la investigación, describir los instrumentos de evaluación y solicitar su permiso. Tras la autorización escolar, se remitió a los padres una carta explicativa del estudio para obtener el consentimiento informado autorizando a sus hijos/as a participar en la investigación. Tras acordar 
la participación de los alumnos, se establecieron, junto con los respectivos tutores, los días para la aplicación de las pruebas, de manera que los sujetos de cada aula cumplimentaran los cuestionarios en sus respectivas aulas en una sola sesión.

Los alumnos fueron informados de la importancia del objeto de estudio y se les invitó a participar de manera voluntaria garantizando la confidencialidad de los datos, obteniendo una buena participación. La evaluación se realizó de igual forma en todos los centros. La batería de pruebas se aplicó el mismo día en horario lectivo, de forma colectiva y voluntaria, asignando a cada alumno un cuadernillo de evaluación. Se indicó que cumplimentaran los datos sociodemográficos y se explicaron las instrucciones de cada una de las pruebas de la batería, destacando la importancia de no dejar respuestas en blanco. Los investigadores que administraron las pruebas verificaron que los individuos contestaron todas las preguntas y aclararon posibles dudas surgidas. El tiempo de aplicación aproximado de la batería de pruebas fue de una hora.

\section{Análisis estadísticos}

Los datos fueron codificados y analizados con el IBM SPSS 22.0. Se realizaron análisis de correlación bivariada y análisis de regresión lineal múltiple con el método "paso a paso" o "por pasos".

\section{RESULTADOS}

Para el análisis de los datos, en primer lugar se comprobó el cumplimiento de los supuestos para ambos modelos: linealidad mediante los gráficos de regresión parcial y de residuos, independencia mediante la prueba Durbin-Watson obteniendo un valor cercano a 2 en ambos casos, homocedasticidad según la homogeneidad de varianzas, normalidad por medio del gráfico P-P y del histograma, y no-colinealidad con una tolerancia próxima a uno en ambos modelos.

Se constató la existencia de correlaciones significativas entre las variables independientes y las variables dependientes (tabla 1). Los resultados indicaron correlaciones positivas significativas entre el índice de rechazos y todos los tipos de ansiedad (excepto TOC), disforia y depresión total, mientras que fueron negativas y significativas con Inteligencia
Social Emocional y con las dimensiones de Empatía y Habilidad Social.

Por su parte el índice de elecciones se asoció positiva y significativamente con Inteligencia Social Emocional, y las dimensiones de Automotivación, Empatía y Habilidad Social, y negativamente con Depresión, Disforia y Autoestima.

Tabla 1.

Correlaciones entre las variables

\begin{tabular}{crcc}
\hline & & $\begin{array}{c}\text { Índice de } \\
\text { rechazos }\end{array}$ & $\begin{array}{c}\text { Índice de } \\
\text { elecciones }\end{array}$ \\
\hline TISE & & $-.33^{* *}$ & $28^{* *}$ \\
& Autoconciencia & -.16 & 13 \\
& Autocontrol & -.19 & 09 \\
& Automotivación & -.19 & $.28^{* *}$ \\
& Empatía & $-.34^{* *}$ & $.21^{*}$ \\
SCAS & Habilidad social & $-.31^{* *}$ & $.29 * *$ \\
& & $.44^{* *}$ & -.17 \\
& & $.34^{* *}$ & -.19 \\
& TAS & $.44^{* *}$ & -.13 \\
& FS & .20 & -.16 \\
& TOC & $.36^{* *}$ & -.15 \\
CDI & TPA & $.33^{* *}$ & -.10 \\
& MDF & $.28^{* *}$ & -.04 \\
& TAG & $.36^{* *}$ & $.28^{* *}$ \\
& & $.46^{* *}$ & $-.24 *$ \\
& Disforia & .14 & $.28^{* *}$ \\
\hline
\end{tabular}

Nota. TISE $=$ Test de Inteligencia Social Emocional; SCAS = Escala de Ansiedad Infantil de Spence; CDI = Inventario de Depresión Infantil; TAS = Trastorno de Ansiedad de Separación; FS = Fobia Social; TOC = Trastorno obsesivo-compulsivo; TPA = Trastorno de Pánico/Agorafobia; MDF = Miedo al Daño físico; TAG $=$ Trastorno de Ansiedad Generalizada.

$* \mathrm{p}<.05 . * * \mathrm{p}<.01$

El modelo predictivo indicó que el índice de rechazos se relacionaba con la sintomatología de fobia social, disforia y baja autoestima, $\mathrm{F}(3,93)=17.68$, $\mathrm{p}=.001$, mientras que el modelo para el índice de elecciones se relacionaba con la variable de habilidades sociales, $\mathrm{F}(1,93)=8.29, \mathrm{p}=.005)$.

Los resultados muestran que el rechazo social se relaciona de manera significativa con la Disforia, la Fobia Social y la Autoestima, explicando un 37\% de la varianza total. El resto de variables fueron excluidas 
del modelo por no resultar significativas dentro del mismo (véase Tabla 2).

Tabla 2

Variables que explican el índice de rechazo social en estudiantes de primaria

\begin{tabular}{|c|c|c|c|c|}
\hline & Constante & $\begin{array}{c}\text { Beta } \\
\text { Estandarizado }\end{array}$ & $\mathbf{t}$ & $\mathbf{p}$ \\
\hline Constante & & & 0.51 & .610 \\
\hline Disforia & & .52 & 4.99 & .000 \\
\hline Fobia Social & & .40 & 4.32 & .000 \\
\hline Autoestima & & -.32 & -3.01 & .003 \\
\hline
\end{tabular}

Nota: R2=.37; Durbin-Watson=1.99; F=17.69; $\mathrm{p}<.01$

La Aceptación Social se relaciona con la variable Habilidad, explicando un $8 \%$ de varianza explicada. El resto de variables fueron excluidas del modelo (Tabla $3)$.

Tabla 3

Variables que explican el índice de aceptación social en estudiantes de primaria

\begin{tabular}{lcll}
\hline Constante & Beta & t & p \\
& Estandarizado & & \\
\hline
\end{tabular}

\begin{tabular}{llll}
\hline Constante & & & \\
Habilidad Social & 0.29 & 2.88 & .005
\end{tabular}

Nota: R2 =. 08; Durbin-Watson = 2.00; F = 8.29; $<<.01$

\section{DISCUSIÓN}

El objetivo principal de este trabajo fue determinar la relación del rechazo/aceptación social con la sintomatología ansioso-depresiva e inteligencia emocional en estudiantes de Primaria. Los resultados evidenciaron que se confirma la primera hipótesis relativa a la relación entre rechazo social y sintomatología ansiosa-depresiva. Así, los alumnos con mayor índice de rechazo social presentaron puntuaciones más altas en las variables clínicas de ansiedad y depresión.

Respecto a la segunda hipótesis, los datos confirman que los alumnos con mayor índice de rechazo social presentaron puntuaciones más bajas en Inteligencia Emocional.
Los resultados de estas dos primeras hipótesis son consistentes con los del estudio de Gallardo y Jiménez (1997), donde los alumnos rechazados muestran más síntomas de ansiedad y depresión, un menor autoconcepto positivo y un mayor autoconcepto negativo, y más problemas de conducta que los alumnos aceptados. Estudios más recientes señalan que sufrir acoso escolar en la infancia tiene un gran impacto en la salud psicológica de los adolescentes que han vivido esas situaciones. Las víctimas de acoso escolar en su niñez presentaban mayores niveles de ansiedad social, de hostilidad y de ansiedad generalizada, junto con menor grado de autoestima, al compararse con aquellas que no habían sufrido acoso (Pradhan, 2006). El perfil psicosocial de los adolescentes que se muestran como víctimas de rechazo social o agresores reflejan, por un lado, tasas más bajas de autoestima general y satisfacción con la vida, y por otro, tasas más elevadas de sintomatología depresiva (Povedano, Estévez, Martínez y Monreal, 2012).

Por otra parte, respecto a las hipótesis 3 y 4 , los resultados muestran la relación existente entre ser elegido por los iguales (aceptación social) y la puntuación en habilidades sociales, por lo que tan solo resultan confirmadas parcialmente. Aun así, estos resultados son coherentes con los de estudios previos que indican que los niños y adolescentes que tienen más amigos, son más competentes socialmente, más cooperativos, presentan menos dificultades de relación con los demás y una autoestima más elevada que se relaciona con mayores índices de bienestar y adaptación psicosocial (Cava y Musitu, 2000).

En general, nuestros resultados contribuyen a lograr una descripción más detallada de las variables que están relacionadas con el rechazo y la aceptación social infantil. Se pone de manifiesto que ser rechazado por los iguales lleva asociada una serie de resultados negativos que incluyen aspectos como síntomas de ansiedad (especialmente fobia social), depresión, baja autoestima, ser menos elegidos para trabajar o jugar por sus compañeros. Estos aspectos son considerados como factores de alto riesgo para presentar alguna psicopatología en el futuro.

Una de las implicaciones más claras y directas de nuestros hallazgos es que apuntan hacia la necesidad 
de desarrollar estrategias para promover ambientes escolares facilitadores de la prevención del rechazo social y de incluir acciones dirigidas a intervenir en las variables identificadas asociadas al rechazo social en este estudio (Valdés y Martínez, 2014) y que esta problemática sea abordada desde una enfoque multicausal, que no sólo se limite a las conductas de los sujetos y a la falta de habilidades sociales o competencia social, que en este caso solo explican un $8 \%$ de la aceptación social y no tienen implicación en el rechazo social, sino que se consideren intervenciones en los diferentes niveles en que se desarrolla el estudiante, prestando atención a los aspectos curriculares y organizativos que favorecen la integración de ellos (Ortega y Del Rey, 2008; Swearer et al., 2009).

Por esto, es fundamental la labor del centro escolar, como agente educador de sus alumnos, ofreciendo un entorno de aprendizaje y convivencia(Calvo y Ballester, 2007). Además cabe mencionar que los programas de convivencia escolar promovidos por las instituciones educativas se ven favorecidos por la posibilidad de coordinar las acciones de los diferentes actores involucrados en la problemática, como los estudiantes, docentes, padres de familia y la comunidad en general (Epstein, Galindo y Sheldon, 2011). Esta investigación sirve como guía para informar a los Centros Escolares sobre características cognitivas y conductuales que pueden predisponer a los alumnos a ser víctimas de rechazo social (Jaramillo et al. 2006). En este sentido se sugiere el desarrollo y mejora de las competencias socio-emocionales en niños para prevenir desde temprana edad el surgimiento del rechazo social y en consecuencia, las patologías asociadas a este, mediante programas de prevención cognitivo-conductuales que han demostrado su eficacia (Mateu-Martínez et al., 2013).

$\mathrm{Si}$ bien los resultados obtenidos en esta investigación son satisfactorios, se necesitan más investigaciones específicas al respecto teniendo en cuenta las limitaciones encontradas. Primero, el Test de Inteligencia Emocional presenta ciertas dificultades respecto a la comprensión del vocabulario, siendo recomendable su validación y adaptación en población española. Segundo, la muestra de esta investigación es reducida, siendo recomendable ampliar el número de participantes para realizar una réplica de este estudio piloto, de manera que se vea favorecida la generalización de los resultados. Tercero, además de los propios sujetos, es conveniente contar con más fuentes de información como padres y profesores favorecería la comparación de los resultados en algunas pruebas utilizadas que cuentan con versión para padres. Cuarto, el estudio tiene las limitaciones propias de los estudios transversales, que no permiten establecer causalidad entre las variables estudiadas (Hernández-Ávila, 2007).

Las líneas futuras apuntan hacia tres vías principalmente. Por un ladoel estudio de las propiedades psicométricas del Test de Inteligencia Social Emocional, realizando una validación y adaptación del cuestionario para población española. Por otro, replicar el estudio ampliando el número de participantes con vistas a la generalización de resultados, contando con más fuentes de información mediante las versiones para padres y profesores de algunos de los cuestionarios, favoreciendo la comparación de los resultados, y tener en cuenta las medidas cualitativas de la elección y el rechazo social. Finalmente, es necesario desarrollar y evaluar la eficacia de programas de prevención del rechazo social, con el fin de promover la aceptación social, mejorar las relaciones entre pares, y prevenir psicopatologías asociadas, como Ansiedad y Depresión en población latinoamericana.

\section{REFERENCIAS}

Albores-Gallo, L., Sauceda-García, J. M., RuizVelasco, S. y Roque-Santiago, E. (2011). El acoso escolar (bullying) y su asociación con trastornos psiquiátricos en una muestra de escolares en México. Salud Pública de México, 53(3), 220227.

Analitis, F., Klein, M., Detmar, S., Erhart, M., RavensSieberer, U., Herdman, M., Berra, S., Alonso, J. y Rajmil, L (2009). Víctimas de acoso: factores asociados en niños y adolescentes de 8-18 años de edad en 11 países europeos. Pediatrics, 67(2), 111-118.

Becerra, S., Godoy, M. P., Véjar, M. y Vidal, N. (2013). Violencia y conductas de rechazo social en la escuela: sus efectos en el estudiante indígena. Teoria e Prática da Educação, 16(2), 9-18. 
Bierman, K. L. (2004). Peer rejection. Developmental, processes and intervention strategies. Nueva York: Guilford Press.

Calvo, A. R. y Ballester, F. (2007). Acoso Escolar: Procedimientos de Intervención. Madrid: EOS.

Cava, M.J. y Musitu, G. (2000). La potenciación de la autoestima en la escuela. Barcelona: Paidós.

Chiriboga, R. D. y Franco, J. E. (2001). Validación de un test de Inteligencia Emocional en niños de 10 años de edad. Revista Médico de Familia, 9(1), 24-38.

Del Barrio, V., Moreno-Rosset, C. y López-Martínez, R. (1999). The Children's Depression Inventory, (CDI; Kovacs, 1992). Su aplicación en población española. Revista Clínica y Salud, 10(3), 393416.

Díaz-Aguado, M. (1995). Niños con dificultades socioemocionales. Instrumentos de evaluación. Madrid: Ministerio de Asuntos Sociales.

Díaz-Aguado, M. (2005). Prevenir la violencia contra las mujeres: construyendo la igualdad. Programa para educación secundaria. Madrid: Instituto de la Mujer.

Epstein, J., Galindo, C. y Sheldon, S. (2011). Levels of leadership: Effects of district and school leaders on the quality of school programs of family and community involvement. Educational Administration Quarterly, 47(3), 462-495.

Estévez, E., Martínez, B. y Jiménez, T.I. (2009). Las relaciones sociales en la escuela: El problema del rechazo escolar. Revista de los Psicólogos de la Educación, 15(1), 45-60.

Fernández, P. y Extremera, N. (2002). La inteligencia emocional como una habilidad esencial en la escuela. Revista Iberoamericana de Educación, 29(1), 1-6.

Gallardo, J. A. y Jiménez, M. (1997). Efectos del maltrato y del status sociométrico sobre la adaptación social y afectiva infantil. Psicothema, 9(1), 119-131.

Gifford-Smith, M. E. y Brownell, C. A. (2003). Childhood peer relationships: Social acceptance, friendships, and peer networks. Journal of School Psychology, 41(4), 235-284.
García-Bacete, F. J., Jiménez, I., Muñoz, M. V., Monjas, M. I., Sureda, I., Ferrá, P. y Sanchiz, M. L. (2013). Aulas como contextos de aceptación y apoyo para integrar a los alumnos rechazados. Apuntes de Psicología, 31(2), 145-154.

García-Bacete, F. J., Monjas, I. y Sureda, M. I. (2008). Distribución sociométrica en las aulas de chicos y chicas a lo largo de la escolaridad. Revista de Psicología Social, 23(1), 63-74.

Goleman, D. (1996) La Inteligencia Emocional. Barcelona: Kairós.

Hernández-Ávila, M. (2007). Epidemiología: diseño y análisis de estudios. Mexico,D.F., Estados Unidos de México: Editorial Médica Panamericana.

Jaramillo, J. M., Díaz, K., Niño, L. A., Tavera, A. L. y Velandia, A. (2006). Factores individuales, familiares y escolares asociados a la aceptación y el rechazo social en grupos de niños escolarizados entre los 9 y 11 años de edad. Revista Diversitas, 2(2), 205-215.

Malinosky-Rummel, R. y Hansen, D. (1993). Longterm consequences of childhood physical abuse. Psychological Bulletin, 114(1), 68-79.

Martínez, J., Ganem, A. E., Contreras, M. M., Leal, E. K., Soto, M. y Fernández, J. (2014). Prevalencia y factores de riesgo para ser víctima de bullying en escolares de 8 a 12 años de edad en una escuela pública. Revista Chilena de Terapia Ocupacional, 14(1), 81-87.

Marande, G. (2011). Estabilidad del rechazo en el primer ciclo de primaria. Trabajo Fin de Máster. Castellón: Universitat Jaume I.

Mateu-Martínez, O., Piqueras, J. A., Jimenez-Albiar, M. I., Espada, J. P., Carballo, J. L. y Orgilés, M. (2013). Eficacia de un programa de prevención cognitivo conductual breve del rechazo social en niños. Terapia Psicológica, 31(2), 187-195.

Figueras, A. Amador-Campos, J. A., Gómez-Benito, J. y del Barrio, V. (2010). Psychometric properties of the Children's Depression Inventory in community and clinical sample. The Spanish Journal of Psychology, 13(2), 990-999.

Mestre, V., Samper, P. y Frías, M. D. (2004). Personalidad y contexto familiar como factores predictores de la disposición prosocial y antisocial 
de los adolescentes. Revista Latinoamericana de Psicología, 36(3), 445-457.

Morison, P. y Masten, A.S. (1991). Peer reputation in middle school as a predictor of adaptation in adolescence: A seven year follow up. Child Development, 62(5), 991-1007.

Moreno, M. C., Muñoz, V., Pérez, P. J. y Sánchez. I. (2004). Incidencia del maltrato entre iguales durante la adolescencia en España. Portularia, 4(4), 307-316.

Orgilés, M., Méndez, X., Spence, S.H.,Huedo-Medina, T. B. y Espada, J. P. (2012). Spanish validation of the Spence Children's Anxiety Scale. Child Psychiatry and Human Development, 43(2), 271281.

Ortega, R. y Del Rey, R. (2008). La violencia escolar. Estrategias de prevención (4ta ed.). Madrid: Grao.

Parker, J. G. y Asher, S. R. (1987). Peer relations and later personal adjustment: Are low-accepted children at risk? Psychological Bulletin, 102(3), 357-389.

Plazas, E. A., Morón Cotes, M. L., Santiago, A., Sarmiento, H., Ariza López, S. E. y Patiño, C. D. (2010). Relaciones entre iguales, conducta prosocial y género desde la educación primaria hasta la universitaria en Colombia. Universitas Psychologica, 9(2), 357-369.

Povedano, A., Estévez, E., Martínez, B. y Monreal, M.C. (2012). Un perfil psicosocial de adolescentes agresores y víctimas en la escuela: Análisis de las diferencias de género. Revista de Psicología Social, 27(2), 169-182.

Pradhan, M. (2006). Adolescent Relational Victimization and Gender Differences in Socialpsychological Adjustment. Michigan: ProQuest.

Seals, D.y Young, J. (2003). Bullying and victimization: prevalence and relationship to gender, grade level, ethnicity, self-esteem, and depression. Adolescence, 38(152), 735-747.

Swearer, S. M., Espelage, D. L. y Napolitano, S. A. (2009). Bullying prevention and intervention: Realistic strategies for schools. Nueva York: Guilford Press.

Valdés, A. y Martínez, E. A. (2014). Relación entre el autoconcepto social, el clima familiar y el clima escolar con el bullying en estudiantes de secundaria. Avances en Psicología Latinoamericana, 32(3), 447-457.
Fecha de recepción: 28 de octubre de 2014

Fecha de aceptación: 10 de noviembre de 2014 